\title{
Baryon and lepton numbers in two scenarios of leptogenesis.
}

\author{
A.Kartavtsev* \\ Institut für Physik, Universität Dortmund, D-44221 Dortmund, Germany
}

\begin{abstract}
Baryon and lepton numbers of the Universe in leptogenesis with Dirac neutrino and leptogenesis with Majorana neutrino scenarios are considered. It is shown that despite quite different features of Dirac and Majorana fermions both scenarios yield the same relation among the initial lepton and the final baryon asymmetries. Moreover right-handed neutrinos in the leptogenesis with Dirac neutrino scenario have very little impact on the effective number of relativistic degrees of freedom, constrained by BBN. Thus the two scenarios are similar from the cosmological point of view. It is also pointed out that in thermal equilibrium the $3 B+L$ sum is zero for left-handed fermions.
\end{abstract}

\section{INTRODUCTION.}

The observed baryon asymmetry of the Universe is one of the most interesting problems of particle physics and cosmology. The cosmological baryon excess can be generated in decays of heavy particles, provided that the three Sakharov conditions [1] are satisfied: baryon (or baryon minus lepton) number is not conserved; $\mathrm{C}$ and $\mathrm{CP}$ are violated; the decay processes are out of equilibrium.

It has been proven to be difficult to generate excess of baryons through direct violation of baryon number. Discovery of anomalous electroweak processes [2], violating baryon $(B)$ and lepton $(L)$ numbers but conserving the difference $B-L$, led to the widely adopted scenario of leptogenesis; the initial lepton number asymmetry, created at a GUT scale, is later converted into baryon asymmetry by anomalous processes (refereed to as sphalerons in what follows). The remaining lepton number is distributed among charged leptons and neutrinos.

According to the scenario suggested by M. Fukugita and T. Yanagida [3] lepton number asymmetry is generated in the lepton number violating and $\mathrm{CP}$ violating decay of heavy Majorana neutrino. After the electroweak $(\mathrm{EW})$ phase transition conventional neutrinos acquire naturally small Majorana masses due to the seesaw mechanism. This scenario is refereed to as leptogenesis with Majorana neutrinos.

In an alternative scenario suggested in [4] total lepton number is conserved, but $\mathrm{CP}$ violating decay of heavy particle results in nonzero lepton number for left-handed particles, and equal in magnitude but opposite in sign lepton number for right-handed particles. The negative lepton number stored in the left-handed neutrinos is converted by the sphalerons into positive baryon number. This scenario is refereed to as leptogenesis with Dirac neutrinos.

These two scenarios are compared here. In particular, the observed baryon asymmetry of the Universe and effects of additional relativistic degrees of freedom are discussed.

The sphaleron process is a crucial ingredient of both

*Electronic address: akartavt@het.physik.uni-dortmund.de scenarios. It is pointed out that if the sphalerons are in thermal equilibrium, then the $3 B+L$ sum is zero for left-handed fermions.

Majorana and Dirac neutrinos have quite different properties. The Dirac neutrino is a four-component fermion and a definite lepton number can be assigned to its left- and right-handed components. The Majorana neutrino is a two-component fermion and the Majorana mass term violates lepton number by two units. Equivalently, the Majorana neutrino does not carry any conserved charges and does not have a chemical potential.

The relation between initial $B-L$ asymmetry generated at the GUT scale and the observed baryon asymmetry of the Universe was studied in [5, 6] using relations among the particles chemical potentials [19]. If the statement about the Majorana neutrino zero chemical potential was applicable in the early Universe, then the initial $B-L$ asymmetry would be completely washed out below the electroweak phase transition. However, the rate of lepton number violating processes, estimated here, turns out to be much smaller than expansion rate of the Universe and thus the neutrino can be assigned an effective chemical potential.

In the scenario of leptogenesis with Dirac neutrino conserved total lepton number is redistributed among the left- and the right-handed components in the processes with Higgs and gauge boson exchange. Due to smallness of the neutrino mass these processes are too slow to bring lepton number of the left- and right-handed neutrinos to equilibrium.

Thus both scenarios yield the same relation between the initial lepton and the final baryon asymmetry. Moreover, since the right-handed neutrino in the scenario of leptogenesis with Dirac neutrino carries only a small fraction of energy density of the Universe, it has very little impact on the effective number of relativistic degrees of freedom, constrained by BBN, and therefore the two scenarios are similar from the cosmological point of view.

\section{ELECTROWEAK PROCESSES.}

Assuming a thermal distribution, the number density of fermions (bosons) of mass $m$ is given by the Fermi- 
Dirac (Bose-Einstein) distribution

$$
n_{ \pm}=\int \frac{\mathrm{d} \mathbf{p}}{(2 \pi)^{3}} \frac{g}{\exp \left[\left(E_{\mathbf{p}} \mp \mu\right) / T\right] \pm 1},
$$

where $\mu$ is the particle chemical potential and $g$ is the number of internal degrees of freedom ( $g=1$ for massless Weyl fermions and $g=2$ for massless vector bosons). Assuming that $\mu / T$ is small and expanding (I) in powers of the particle mass $m$ we find for excess of particles over antiparticles

$$
\begin{aligned}
& n_{+}-n_{-}=\frac{g T^{3}}{6} \frac{\mu}{T} c_{f}\left(\frac{m}{T}\right) \text { (fermions), } \\
& n_{+}-n_{-}=\frac{g T^{3}}{3} \frac{\mu}{T} c_{b}\left(\frac{m}{T}\right) \text { (bosons). }
\end{aligned}
$$

where the functions $c_{f}$ and $c_{b}$ are given by

$$
c_{f}\left(\frac{m}{T}\right) \approx 1-\frac{3}{2 \pi^{2}} \frac{m^{2}}{T^{2}}, c_{b}\left(\frac{m}{T}\right) \approx 1-\frac{9}{2 \pi^{2}} \frac{m}{T}+\frac{3}{4 \pi^{2}} \frac{m^{2}}{T^{2}}
$$

Baryon asymmetry of the Universe, which is usually expressed as ratio of the baryon density to the entropy density of the Universe, is given by

$$
Y_{B}=\frac{n_{B}-n_{\bar{B}}}{s}=(0.6-1) \cdot 10^{-10}
$$

In the radiation dominated Universe the entropy density $s=2 \pi^{2} g_{*} T^{3} / 45$, where $g_{*}$ counts the total number of relativistic degrees of freedom, and therefore $\mu / T \sim Y_{B}$ is sufficiently small to justify the use of eq. (2).

As the sphaleron processes switch off immediately or slightly below the electroweak phase transition, only processes which are in equilibrium in the vicinity of $T_{C} \sim 300$ $\mathrm{GeV}$ are important for baryon and lepton number formation. Success of the standard model (SM) in explaining low-energy phenomena suggests, that all particles besides the known ones are too heavy to be in thermal equilibrium at temperatures close to the critical.

The standard model consists of $N$ generations of quarks and leptons, fields of $S U_{C}(3) \otimes S U_{L}(2) \otimes U_{Y}(1)$ gauge group and $m$ Higgs doublets [20]. Above the electroweak phase transition vacuum expectation value of the Higgs boson is zero and all the known fermions are massless. As temperature drops below the critical one, the fermions acquire masses. In the vicinity of $T_{C}$ the mass to temperature ratio is very small for all fermions but the $t$-quark.

Since the $S U_{C}(3)$ symmetry is exact at any temperature, chemical potentials of the components of color triplets are equal, i.e. chemical potential of the gluon fields is zero.

Above the electroweak phase transition the $S U_{L}(2)$ symmetry is unbroken and components of weak doublets have the same chemical potentials. Therefore chemical potentials of the $W$ bosons are zero. Chemical potential of the $B^{0}$ gauge boson is zero because it is neutral.

Below the phase transition chemical potentials of the components of weak doublets are no longer equal and the
$W^{-}$boson acquires a nonzero chemical potential denoted by $\mu_{W}$. Since the photon and the $Z$ boson are neutral, they do not have chemical potentials.

Rapid flavor-changing interactions in the quark sector assure that chemical potentials of quarks of given charge and chirality are the same for all flavors and colors both above and below the phase transition, so that only four chemical potentials should be introduced: $\mu_{u L}$ and $\mu_{d L}$ for left-handed and $\mu_{u R}$ and $\mu_{d R}$ for right-handed quarks of a given color

In the absence of rapid flavor-mixing interactions chemical potentials $\mu_{i L}$ and $\mu_{i R}$ of charged leptons and chemical potentials $\mu_{i}$ of left-handed neutrinos are in general different. The phenomenon of neutrino oscillation, confirmed experimentally, suggests that below the phase transition flavor-mixing interactions in the neutrino sector may be sufficiently fast to assure $\mu_{i L}=\mu_{L}$, $\mu_{i R}=\mu_{R}$ and $\mu_{i}=\mu_{\nu}$ equalities. Above the phase transition this is, however, not the case. The reason is the following. The source of the light neutrino mass is $\lambda \bar{\nu}_{R} \nu_{L} H$ term. In the case of leptogenesis with Majorana neutrino mass of the right-handed neutrino $\nu_{R}$ is many orders of magnitude bigger than $T_{C}$, and consequently flavor-changing interactions involving this vertex are out of equilibrium. In the case of leptogenesis with Dirac neutrino the coupling $\lambda$ is very small (as is required by the small neutrino mass) and flavor-changing interactions involving the Higgs exchange are out of equilibrium as well.

Finally, it is assumed, that mixing between the Higgs doublets assures the equality of their chemical potentials: $\mu_{-}$for all charged scalars and $\mu_{0}$ for the neutral ones. As the temperature drops below $T_{C}$, the neutral Higgs develops nonzero vacuum expectation value and breaks the $S U_{L}(2)$ symmetry. Since the neutral physical Higgs does not carry any conserved quantum numbers its chemical potential $\mu_{0}$ is zero below the electroweak phase transition.

The electroweak interactions are in thermal equilibrium down to about $T_{d e c} \simeq 2 \mathrm{MeV}$, which imply some useful relations among the chemical potentials [5] partially discussed above.

$$
\begin{aligned}
\mu_{W} & =\mu_{-}+\mu_{0} \quad\left(W^{-} \leftrightarrow H^{-}+H^{0}\right) \\
\mu_{d L} & =\mu_{u L}+\mu_{W} \quad\left(W^{-} \leftrightarrow \bar{u}_{L}+d_{L}\right) \\
\mu_{i L} & =\mu_{i}+\mu_{W} \quad\left(W^{-} \leftrightarrow \bar{\nu}_{i L}+e_{i L}\right) \\
\mu_{u R} & =\mu_{u L}+\mu_{0} \quad\left(H^{0} \leftrightarrow \bar{u}_{L}+u_{R}\right) \\
\mu_{d R} & =\mu_{u L}+\mu_{W}-\mu_{0} \quad\left(H^{0} \leftrightarrow d_{L}+\bar{d}_{R}\right) \\
\mu_{i R} & =\mu_{i}+\mu_{W}-\mu_{0} \quad\left(H^{0} \leftrightarrow e_{i L}+\bar{e}_{i R}\right)
\end{aligned}
$$

Using the relations above, one can express baryon and lepton number, as well as electric charges, in terms of just $3+N$ chemical potentials. Furthermore it is convenient to introduce the following notation:

$$
\begin{aligned}
c_{-} & =c_{b}\left(m_{\phi_{-}} / T\right) \\
c_{W} & =c_{b}\left(m_{W} / T\right) \\
c_{\ell_{i}, u_{i}, d_{i}} & =c_{f}\left(m_{\ell_{i}, u_{i}, d_{i}} / T\right) .
\end{aligned}
$$


The Higgs bosons are massive both above and below the phase transition, and therefore $c_{-}$differs from unity at any temperature 21]. Fermions and gauge bosons are massless above the phase transition, and consequently $c_{W}$ and $c_{\ell_{i}}, c_{u_{i}}, c_{d_{i}}$ are equal to unity at $T>T_{C}$. Deviation from unity at $T<T_{C}$ is small and is parametrized by

$$
\begin{aligned}
N \Delta_{\ell, u, d} & =N-\sum_{i} c_{\ell_{i}, u_{i}, d_{i}}, \\
\Delta_{\mu} & =\mu-\sum_{i} c_{\ell_{i}} \mu_{i},
\end{aligned}
$$

where $\mu \equiv \sum_{i} \mu_{i}$ and $N$ is the number of generations. In the presence of fast flavor-changing interactions in the lepton sector $\Delta_{\mu}=\mu \Delta_{\ell}$.

Chemical potentials $\mu_{i}$ of the neutrinos appear only in the combinations $\mu$ and $\Delta_{\mu}$ and we can express total baryon and lepton numbers in terms of just five chemical potentials. Omitting an overall coefficient we find

$$
\begin{aligned}
B & =N\left(1-\Delta_{u}\right)\left(\mu_{u L}+\mu_{u R}\right)+N\left(1-\Delta_{d}\right)\left(\mu_{d L}+\mu_{d R}\right) \\
& =2 N\left(2-\Delta_{u}-\Delta_{d}\right) \mu_{u L}+2 N\left(1-\Delta_{d}\right) \mu_{W} \\
L & =\sum_{i}\left[\mu_{i}+c_{\ell_{i}}\left(\mu_{i L}+\mu_{i R}\right)\right]=3 \mu-2 \Delta_{\mu} \\
& +2 N\left(1-\Delta_{\ell}\right) \mu_{W}-N\left(1-\Delta_{\ell}\right) \mu_{0}
\end{aligned}
$$

To keep track of the number of different species in the plasma it is useful to introduce electric charges of up and down quarks and charged leptons

$$
\begin{aligned}
Q_{u} & =2 N\left(1-\Delta_{u}\right)\left(2 \mu_{u L}+\mu_{0}\right) \\
Q_{d} & =-N\left(1-\Delta_{d}\right)\left(2 \mu_{u L}+2 \mu_{W}-\mu_{0}\right) \\
Q_{l} & =2 \Delta_{\mu}-2 \mu-2 N\left(1-\Delta_{\ell}\right) \mu_{W}+N\left(1-\Delta_{\ell}\right) \mu_{0}
\end{aligned}
$$

In the massless limit [5] the combined charge of the Higgs and the gauge bosons

$$
Q_{W+H}=-2(m+2) \mu_{W}+2 m \mu_{0}
$$

is given by one and the same formula both above and below the phase transition. When mass effects are taken into account $c_{-}$and $c_{W}$ are not equal and such a representation is no longer possible.

Above the EW phase transition $\mu_{W}=0$ and we obtain

$$
Q_{H+W}=2 m c_{-} \mu_{0}
$$

Below the phase transition two electrically charged components of the Higgs fields are "eaten up" by the $W$. As massive vector boson has three spin degrees of freedom, and $\mu_{0}$ is zero at this stage, the expression for the combined charge takes the form

$$
Q_{H+W}=-2\left((m-1) c_{-}+3 c_{W}\right) \mu_{W}
$$

In the case of the standard model $(m=1)$ the physical Higgs is neutral and only the $W$-boson contributes to the charge density.

\section{SPHALERON PROCESSES.}

An important ingredient of leptogenesis is the electroweak anomaly which violates $B$ and $L$ but conserves $B-L$ quantum number. Let us first consider the case of only left-handed fermions above the electroweak phase transition. As the chemical potentials of up and down components of electroweak doublets are equal and fermion masses are zero, the free energy $\mathcal{F}$ is given by

$$
\mathcal{F}=2 \sum_{i} F\left(\mu_{i}\right)+6 N F\left(\mu_{u L}\right) \propto 2 T^{2} \sum_{i} \mu_{i}^{2}+6 T^{2} N \mu_{u L}^{2}
$$

Baryon and lepton number densities are obtained by differentiating the free energy with respect to $\mu_{i}$ and $\mu_{u L}$

$$
L_{L}=\sum_{i} \frac{\partial \mathcal{F}}{\partial \mu_{i}} \propto 4 T^{2} \mu, \quad B_{L}=\frac{1}{3} \frac{\partial \mathcal{F}}{\partial \mu_{u L}} \propto 4 T^{2} N \mu_{u L} .
$$

From the fact that the sphalerons conserve baryon minus lepton quantum number separately for each generation 7] it follows that $d \mu_{i}=d \mu_{u L}$. Minimization of the free energy yields

$$
\frac{d \mathcal{F}}{d \mu_{u L}} \propto 4 T^{2}\left(3 N \mu_{u L}+\mu\right) \propto 3 B_{L}+L_{L}=0
$$

In other words, if sphaleron processes are in equilibrium sum of the lepton and thrice the baryon number stored in the left-handed fermions is zero.

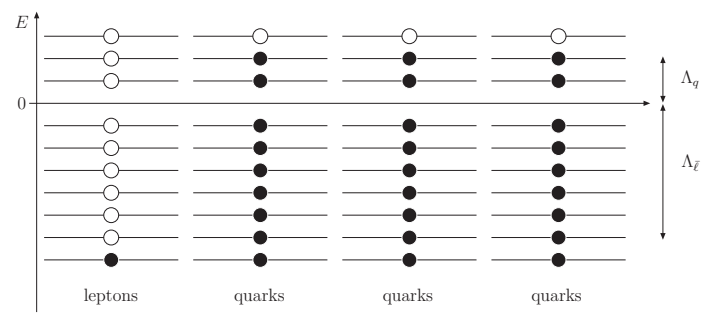

FIG. 1: Illustration to conversion of lepton number into baryon number by the electroweak anomaly. $\Lambda_{\bar{\ell}}$ and $\Lambda_{q}$ are (positive) maximal energies of antileptons and quarks.

To illustrate this conclusion let us assume that initial baryon number is zero and total lepton number is negative, as it is the case for leptogenesis. Number and energy densities of leptons and quarks are given by

$$
n_{\bar{\ell}} \propto \Lambda_{\bar{\ell}}^{3}, \quad n_{q} \propto 3 \Lambda_{q}^{3}, \quad E_{\bar{\ell}} \propto \Lambda_{\bar{\ell}}^{4}, \quad E_{q} \propto 3 \Lambda_{q}^{4}
$$

In the course of sphaleron transitions empty negative lepton levels and filled negative quark levels cross the zero from below so that number of antileptons is decreased and number of baryons is increased (Fig. 1). While both $\Lambda_{\bar{\ell}}$ and $\Lambda_{q}$ change, the sum $\Lambda_{\bar{\ell}}+\Lambda_{q}$ remains constant.

$$
\frac{d E}{d \Lambda_{q}} \propto 3 \Lambda_{q}^{3}-\Lambda_{\bar{\ell}}^{3} \propto n_{q}-n_{\bar{\ell}} \propto 3 B_{L}+L_{L}=0
$$

The energy density of the system reaches its minimum when total number of antileptons is equal to total number 
of quarks, i.e. the baryon number is minus one third of the lepton number.

In a more general case it is convenient to use the fact that for equilibrium reactions sum of chemical potentials of the incoming particles is equal to that of outgoing. The sphaleron processes correspond to the creation of $\left(u_{i L} d_{i L} e_{i L} \nu_{i L}\right)$ states of each generation [7] out of the vacuum [22]. Therefore, as long as the sphaleron processes are in thermal equilibrium, the following relation among the chemical potentials is enforced:

$$
3 N\left(\mu_{u L}+\mu_{d L}\right)+\sum_{i}\left(\mu_{i L}+\mu_{i}\right)=0,
$$

where the factor of three is due to the three color degrees of freedom, while summation over generations takes into account that fermions of all the generations are created simultaneously. Relations (5) allow to rewrite (11) as follows:

$$
3 N \mu_{u L}+2 N \mu_{W}+\mu=0 .
$$

Above the electroweak phase transition (i.e. in the limit of $\left.\mu_{W}=0\right)$ the last equality coincides with the eq. (10).

\section{ABOVE THE PHASE TRANSITION.}

Above the phase transition the left-handed neutrino is a massless Weyl fermion in both scenarios. Since the gauge bosons and the fermions are massless, the anomalous electroweak processes are not suppressed. As the chemical potential of the $W$ is zero and $\Delta_{\mu}=0$ baryon and lepton numbers are proportional to the total $B-L$ asymmetry. Requiring that total electric charge $Q$ is zero and making use of the relation implied by sphaleron transitions we find $[5,6]$

$$
\begin{aligned}
& B=\frac{8 N+4 m c_{-}}{22 N+13 m c_{-}}(B-L) \approx 0.36(B-L), \\
& L=-\frac{14 N+9 m c_{-}}{22 N+13 m c_{-}}(B-L) \approx-0.64(B-L),
\end{aligned}
$$

where for the numerical evaluation we shall choose $N=3$ and assume only one Higgs doublet with the Higgs mass of $400 \mathrm{GeV}$ at $T_{C}$. While the $3 B+L$ number stored in left-handed particles is zero, neither total $3 B+L$ nor total $B+L$ are zero.

Electric charges stored in different species are given by

$$
\begin{aligned}
Q_{u} & =\frac{4 m c_{-}}{22 N+13 m c_{-}}(B-L) \approx 0.03(B-L) \\
Q_{d} & =-\frac{8 N+2 m c_{-}}{22 N+13 m c_{-}}(B-L) \approx-0.34(B-L) \\
Q_{l} & =\frac{8 N+6 m c_{-}}{22 N+13 m c_{-}}(B-L) \approx 0.37(B-L)
\end{aligned}
$$

i.e., above the electroweak phase transition there is an excess of down quarks and total electric charge of the leptons is positive.

\section{LEPTOGENESIS WITH DIRAC NEUTRINOS.}

According to the scenario suggested in 4], even in a model that conserves lepton number, a $\mathrm{CP}$ violating decay of heavy particle can result in a nonzero lepton number for left-handed particles, and equal in magnitude but opposite in sign lepton number for right-handed particles. The negative lepton number stored in the lefthanded fermions is later converted into positive baryon number by the sphalerons .

In this scenario below the phase transition the neutrino is a four-component Dirac spinors with very small mass. Coupling of its right-handed component to the neutral Higgs is given by

$$
\mathcal{L}=\frac{g}{2} \frac{m_{\nu}}{M_{W}} \bar{\nu} \nu H_{0}
$$

In (15) the Yukawa coupling is expressed as a ratio of the neutrino mass to the Higgs VEV. The later, in turn, is proportional to the $W$ mass.

Negative and positive lepton numbers stored, respectively, in the left- and right-handed neutrinos can partially equilibrate. However, as is clear from (15), rate of the equilibration processes is strongly suppressed by the neutrino to the $W$ mass ratio. To be more quantitative, let us evaluate the corresponding diagrams. In the processes depicted in Fig. 2 (and their charge conjugate) total lepton number is conserved, but lepton asymmetry is redistributed among the left- and right-handed neutrinos.

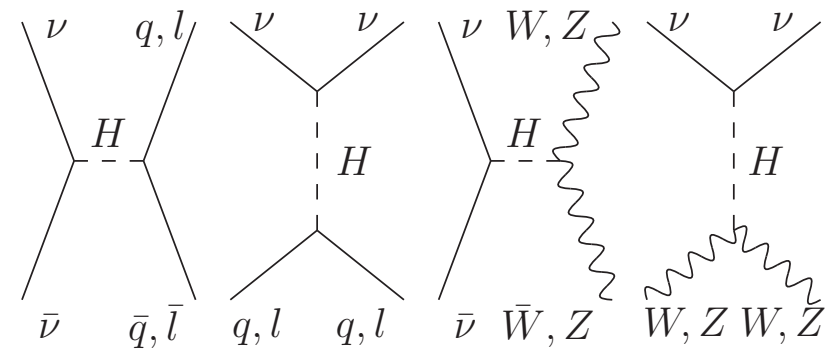

FIG. 2: Higgs-mediated processes which equilibrate lepton asymmetry stored in the left- and right-handed neutrinos.

The leading contribution at $T \sim T_{C}$ is due to the topquark, whose Yukawa coupling is of order of unity, and due to the $W$ and $Z$ bosons. Reduced cross sections of the diagrams with the top-quark are given by

$$
\begin{aligned}
& \hat{\sigma}_{s}^{t}=\frac{g^{4}}{32 \pi}\left(\frac{m_{\nu}}{M_{W}} \frac{m_{t}}{M_{W}}\right)^{2} \frac{\sqrt{z}(z-4)^{1.5}}{(z-a)^{2}} \\
& \hat{\sigma}_{t}^{t}=\frac{g^{4}}{32 \pi}\left(\frac{m_{\nu}}{M_{W}} \frac{m_{t}}{M_{W}}\right)^{2} \int_{0}^{y_{f}} \frac{z y(z y+4)}{(z y+a)^{2}} d y
\end{aligned}
$$

where $z \equiv s / m_{t}^{2}$ and $a \equiv m_{H}^{2} / m_{t}^{2}$ are introduced, and the limit of integration $y_{f}=\left(1-m_{t}^{2} / s\right)^{2}$. 
Reduced cross sections of the diagrams with the $W$ boson in the initial and final states are given by

$$
\begin{aligned}
& \hat{\sigma}_{s}^{W}=\frac{g^{4}}{16 \pi}\left(\frac{m_{\nu}}{M_{W}}\right)^{2} \frac{\left(3-z+z^{2} / 4\right) \sqrt{z(z-4)}}{(z-b)^{2}} \\
& \hat{\sigma}_{t}^{W}=\frac{g^{4}}{16 \pi}\left(\frac{m_{\nu}}{M_{W}}\right)^{2} \int_{0}^{y_{g}} \frac{\left(3+z y+z^{2} y^{2} / 4\right) z y d y}{(z y+b)^{2}}
\end{aligned}
$$

where $z \equiv s / M_{W}^{2}$ and $b \equiv m_{H}^{2} / M_{W}^{2}$ are introduced, and the limit of integration $y_{g}=\left(1-M_{W}^{2} / s\right)^{2}$. In the case of processes with the $Z$ boson $g$ and $M_{W}$ are to be replaced by $\bar{g}$ and $M_{Z}$ respectively. In addition, overall coefficient of expression for the $s$-channel reduced cross section $\hat{\sigma}_{s}^{Z}$ is factor of two smaller than that in (18).

At the temperature range of interest $T \geqslant m_{H, W, Z, q, \ell}$ and approximate analytical expressions for the reaction densities can be obtained. To leading order

$$
\begin{gathered}
\gamma_{s}^{t} \approx \gamma_{t}^{t} \approx \frac{g^{4} T^{4}}{512 \pi^{5}}\left(\frac{m_{\nu}}{M_{W}} \frac{m_{t}}{M_{W}}\right)^{2} \\
\gamma_{s}^{W} \approx 2 \gamma_{t}^{W} \approx 2 \gamma_{s}^{Z} \approx 2 \gamma_{t}^{Z} \approx \frac{g^{4} T^{4}}{128 \pi^{5}}\left(\frac{m_{\nu}}{M_{W}} \frac{T}{M_{W}}\right)^{2}
\end{gathered}
$$

where the tree-level relation $g M_{Z}=\bar{g} M_{W}$ was used.
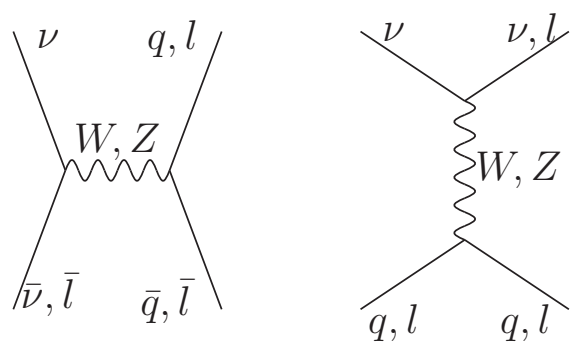

FIG. 3: Gauge-mediated processes which equilibrate lepton asymmetry stored in the left- and right-handed neutrinos. Here $l$ stands for both charged leptons and neutrinos.

Diagrams with exchange of the gauge bosons depicted in Fig. [3] also contribute to equilibration of lepton asymmetry stored in the left- and right-handed neutrinos. As right-handed neutrino scattering mediated by a gauge boson is a helicity flipping process, its amplitude is proportional to the neutrino mass. Unless the second lepton in the same vertex is also right-handed (amplitude of such a process is further suppressed by charged lepton or neutrino mass), helicity of a particle in the second vertex is flipped as well, so that the amplitude is proportional to mass of this particle. Therefore, the leading contribution at the temperature range of interest is due to heavy quarks, in particular the $t$-quark. In the case of charged current

$$
\begin{aligned}
& \hat{\sigma}_{s}^{c c} \approx \frac{g^{4}}{32 \pi}\left(\frac{m_{\nu}}{M_{W}} \frac{m_{t}}{M_{W}}\right)^{2} \frac{(z-c)^{2}}{(z-1)^{2}} \\
& \hat{\sigma}_{t}^{c c} \approx \frac{g^{4}}{32 \pi}\left(\frac{m_{\nu}}{M_{W}} \frac{m_{t}}{M_{W}}\right)^{2} \int_{0}^{y_{f}} \frac{z y(z y+c)}{(z y+1)^{2}}
\end{aligned}
$$

where $c \equiv m_{t}^{2} / M_{W}^{2}$ and $y_{f} \equiv\left(1-m_{t}^{2} / s\right)$ are introduced. In the case of neutral current $g$ and $M_{W}$ are to be replaced by $\bar{g}$ and $M_{Z}$ respectively. In addition, overall coefficient of the expressions for reduced cross sections is factor of two smaller than that in (22) and (23), $a=0$ and the limit of integration $y_{f}=\left(1-m_{t}^{2} / s\right)^{2}$. At the temperature range of interest the corresponding reaction densities are given to leading order by

$$
\gamma_{t}^{c c} \approx \gamma_{s}^{c c} \approx 2 \gamma_{s}^{n c} \approx 2 \gamma_{s}^{n c} \approx \frac{g^{4} T^{4}}{512 \pi^{5}}\left(\frac{m_{\nu}}{M_{W}} \frac{m_{t}}{M_{W}}\right)^{2}
$$

Right-hand side of the Boltzmann equations in the expanding Universe [8] is proportional to the $x \gamma / s H$ ratio. As sphalerons are active only at temperatures $T \gtrsim M_{W}$, it is convenient to define $x=M_{W} / T$, so that $x$ is of order of unity in the relevant range of temperatures. Then the ratios are given by

$$
\begin{gathered}
\frac{x \gamma_{s}^{t}}{s H} \approx \frac{x \gamma_{t}^{t}}{s H} \approx \frac{x \gamma_{s}^{c c}}{s H} \approx \frac{x \gamma_{t}^{c c}}{s H} \approx 2 \frac{x \gamma_{s}^{n c}}{s H} \approx 2 \frac{x \gamma_{t}^{n c}}{s H} \sim 10^{-13} \\
\frac{x \gamma_{s}^{W}}{s H} \approx 2 \frac{x \gamma_{t}^{W}}{s H} \approx 2 \frac{x \gamma_{s}^{Z}}{s H} \approx 2 \frac{x \gamma_{t}^{Z}}{s H} \sim 10^{-13}\left(\frac{T}{M_{W}}\right)^{2}
\end{gathered}
$$

where for the numerical evaluation we used $m_{\nu} \sim 1 \mathrm{eV}$. The reaction densities are clearly extremely small and therefore the lepton number asymmetry does not come to equilibrium and total number of the right-handed neutrinos carrying positive lepton number remain (almost) constant in time.

Neutrino mass eigenstates are related to the weak interaction flavor eigenstates by a nondiagonal unitary transformation. Provided that oscillations induced by the neutrino mixing are in thermal equilibrium, chemical potentials of different neutrino species are equal. Analysis of the neutrino oscillations in the early universe has been performed in [9, 10] where it was concluded that the oscillations begun at about $T \simeq 30 \mathrm{MeV}$ and flavor equilibrium was achieved before the big-bang nucleosynthesis (BBN) epoch. At temperatures just below the critical one the oscillations are effectively frozen, and the neutrino chemical potentials $\mu_{i}$ are in general not equal, so that $\Delta_{\mu}$ differs from zero. Conservation of $B-L$ for each family separately (together with the rapid flavor-mixing interactions in the quark sector, which assure even distribution of baryon number among generations) allows to express $\Delta_{\mu}$ in terms of individual lepton numbers $L_{i}$ [23]. From (6a) and (6b) it follows that below the electroweak phase transition

$$
\begin{aligned}
\left(1+2 c_{\ell_{i}}\right) \mu_{i} & =\left(4-2 \Delta_{u}-2 \Delta_{d}\right) \mu_{u L} \\
& +\left(2-2 c_{i \ell}-2 \Delta_{d}\right) \mu_{W}-\left(B / N-L_{i}\right)
\end{aligned}
$$

Expanding $\mu_{i}$ and $c_{\ell_{i}} \mu_{i}$ in powers of $1-c_{\ell_{i}}$ and keeping only terms linear in $\Delta_{\ell, u, d}$ we obtain to leading order

$$
\begin{aligned}
3 \Delta_{\mu} & =\left[4 N \mu_{u L}-(B-L)\right] \Delta_{\ell}-\Delta, \\
\Delta & =\sum_{i}\left(L / N-L_{i}\right)\left(1-c_{\ell_{i}}\right)
\end{aligned}
$$


Requiring that total electric charge is zero and assuming that the sphaleron processes are still in equilibrium 7] immediately after the phase transition 24] we find:

$$
\begin{aligned}
B & =\frac{8 N+4 \bar{m}}{24 N+13 \bar{m}}(B-L)+\Delta B \\
& \approx 0.32(B-L)+0.59 \Delta, \\
L & =-\frac{16 N+9 \bar{m}}{24 N+13 \bar{m}}(B-L)+\Delta L \\
& \approx-0.68(B-L)+0.59 \Delta, \\
\Delta B & =\Delta L \equiv \frac{14 N+8 \bar{m}}{24 N+13 \bar{m}} \frac{\Delta}{3},
\end{aligned}
$$

where $\bar{m}=(m-1) c_{-}+3 c_{W}$ is introduced. In comparison with the massless case [5] the correction due to the Higgs and $W$ mass is about a factor of two. Correction due to the fermion masses is about $5 \%$ and neglected in (29a) and (29b). In other words, to obtain relations (29) we neglect $\Delta_{\ell, u, d}$ but keep $\Delta$ and $\Delta_{\mu}$ different from zero in equations (6), (7) and (28). For the numerical evaluation we use $N=3$ and assume only one Higgs doublet. For definiteness the sphaleron freeze out temperature is chosen to be equal to the $W$ mass.

To the same accuracy electric charges of different species are given by

$$
\begin{aligned}
Q_{u} & =\frac{4 \bar{m}}{24 N+13 \bar{m}}(B-L)+\frac{24 N+8 \bar{m}}{24 N+13 \bar{m}} \frac{\Delta}{3} \\
& \approx 0.07(B-L)+0.30 \Delta, \\
Q_{d} & =-\frac{8 N+2 \bar{m}}{24 N+13 \bar{m}}(B-L)-\frac{2 N+4 \bar{m}}{24 N+13 \bar{m}} \frac{\Delta}{3} \\
& \approx-0.28(B-L)-0.04 \Delta, \\
Q_{l} & =\frac{8 N+6 \bar{m}}{24 N+13 \bar{m}}(B-L)-\frac{22 N+14 \bar{m}}{24 N+13 \bar{m}} \frac{\Delta}{3} \\
& \approx 0.36(B-L)-0.32 \Delta .
\end{aligned}
$$

If $B-L$ is large in comparison with $\Delta$, then there is an excess of down quarks and total electric charge of the leptons is positive immediately after the phase transition. On the contrary, if $B-L$ is small there is an excess of up quarks and the total charge of the leptons is negative.

Even if the total $B-L$ asymmetry is zero, resulting baryon $(\Delta B)$ and lepton $(\Delta L)$ numbers are nonzero due to the mass effects. Let us assume for a moment, that the total $B-L$ (more precisely, total initial lepton number of the left-handed fermions in the scenario under consideration) is zero and the final baryon and lepton numbers are nonzero only due to the mass effects. Given the smallness of the charged lepton masses, what are the $L_{i}$ needed to reproduce the observed baryon asymmetry of the Universe? Taking for the fermion masses their values at zero temperature (which is expected to be a good approximation if the phase transition is of the first order) we find

$$
\begin{aligned}
B=L & \approx \mathcal{O}(0.1) \cdot\left[10^{-6}\left(L / N-L_{\tau}\right)\right. \\
& \left.+10^{-8}\left(L / N-L_{\mu}\right)+10^{-13}\left(L / N-L_{e}\right)\right]
\end{aligned}
$$

From (31) it immediately follows that deviation of individual leptons numbers from zero should be at least seven orders of magnitude bigger than the observed baryon asymmetry. Large individual lepton numbers $\left|L_{i}\right| \gg 10^{-9}$ mean excess of energy density of neutrino sea and lead to an increased expansion rate for the Universe which subsequently allows less time for the neutrons to decay into protons [9]. However, as temperature drops down to $T_{\text {osc }} \simeq 30 \mathrm{MeV}$ the oscillations equalize individual asymmetries (chemical potentials) of the neutrinos. Therefore chemical potential of the electron neutrino at the BBN epoch is determined only by the total baryon and lepton numbers.

Let us illustrate this conclusion by estimating the neutrino chemical potential which is constrained by BBN and CMBR/LSS to be in the range 11]

$$
-0.01<\mu_{\nu} / T<0.22,
$$

and the difference $\mu_{e}-\mu_{\nu}$ which enters into expression for the neutron to proton ratio. The only leptons at this stage are the electron and neutrinos. Ratio of the electron mass to temperature is still small and we therefore neglect deviation of $c_{e}$ from unity. Lepton number and electric charge are given by

$$
Y_{L}=\frac{15}{4 \pi^{2} g_{*}^{b b n}}\left(N \frac{\mu_{\nu}}{T}+2 \frac{\mu_{e}}{T}\right), Y_{Q}=-\frac{15}{4 \pi^{2} g_{*}^{b b n}}\left(2 \frac{\mu_{e}}{T}\right),
$$

where $g_{*}^{b b n}=10.75$ is the effective number of relativistic degrees of freedom which are in thermal equilibrium with the plasma at the BBN epoch. Baryons are in form of neutrons and protons at this stage:

$$
Y_{B}=Y_{p}+Y_{n} \equiv(1+r) Y_{p}
$$

where the neutron to proton ratio $r \approx 1 / 7$. Charge neutrality of the Universe implies $Y_{Q}+Y_{p}=0$, and we obtain for the chemical potentials

$$
\begin{aligned}
\frac{\mu_{\nu}}{T} & =\frac{4 \pi^{2} g_{*}^{b b n}}{15 N}\left(Y_{L}-Y_{p}\right) \\
\frac{\mu_{e}-\mu_{\nu}}{T} & =\frac{4 \pi^{2} g_{*}^{b b n}}{30 N}\left((N+2) Y_{p}-2 Y_{L}\right)
\end{aligned}
$$

As follows from (29), both $Y_{L}$ and $Y_{B}$ are of order of $10^{-9}$ and therefore the BBN constrain (32) is certainly satisfied. The difference $\mu_{e}-\mu_{\nu}$ is very small as well, so that the canonical result for the neutron to proton ratio remains unaffected.

Right-handed neutrinos decouple at a temperature which is well above the temperature of the electroweak phase transition. At this stage all the SM degrees of freedom are relativistic and new, yet unknown, particle species are very likely to be relativistic as well. Standard analysis [8] yields an upper limit for the additional effective number of neutrino degrees of freedom at the BBN epoch

$$
\Delta N_{\nu}=N\left(g_{*}^{b b n} / g_{*}^{g u t}\right)^{\frac{4}{3}} \leqslant 0.13
$$


consistent with the BBN constrain $\Delta N_{\nu} \leqslant 0.2$ [12]. For numerical evaluation we used $g_{*}^{g u t}=112$ - effective number of relativistic degrees of freedom in the standard model supplemented by three light right-handed neutrinos. In extensions of the $\mathrm{SM} \Delta N_{\nu}$ is even smaller.

Below the electroweak decoupling temperatures of the left- and right-handed neutrinos scale as

$$
T_{\nu_{L}}=\left(g_{*} / g_{*}^{d e c}\right)^{\frac{1}{3}} T, \quad T_{\nu_{R}}=\left(g_{*} / g_{*}^{g u t}\right)^{\frac{1}{3}} T
$$

where the effective number of relativistic degrees of freedom at the electroweak decoupling $g_{*}^{d e c}=11 / 2$. Ratio of the two temperatures

$$
T_{\nu_{R}} / T_{\nu_{L}}=\left(g_{*}^{\text {dec }} / g_{*}^{g u t}\right)^{\frac{1}{3}} \leqslant 0.36
$$

The left-handed neutrino become nonrelativistic as temperature drops down to $T=(11 / 4)^{\frac{1}{3}} m_{\nu} \approx 1.4 m_{\nu}$. The right-handed one becomes nonrelativistic at a higher temperature $T \simeq 3.9 m_{\nu}$. As the neutrino components are effectively decoupled from the other species, their energy density is not transfered to other particles and therefore energy distribution of the nonrelativistic neutrinos deviates from the thermal one.

\section{LEPTOGENESIS WITH MAJORANA NEUTRINOS.}

In the scenario of leptogenesis suggested M. Fukugita and T. Yanagida [3] CP-violating decay of heavy Majorana neutrino is the source of the initial $B-L$ asymmetry. After the EW phase transition the conventional neutrinos receive small Majorana masses via the see-saw mechanism, i.e. conventional neutrino is a two component Majorana fermion in this scenario.

As Majorana fermions do not carry any conserved quantum numbers, strictly speaking they do not have a chemical potential. If this statement was applicable in the early Universe, baryon and lepton number asymmetries generated above the electroweak phase transition would be completely washed out below the phase transition [25].

For a massless fermion helicity and associated lepton number are conserved quantities. Presence of the Majorana mass term means that there are processes which flip helicity and violate lepton number by two units [26]. Rate of such processes is proportional to the neutrino mass and, given the current experimental limits [13, 14], is smaller than the expansion rate of the universe. In what follows we assign [15] positive lepton number to neutrinos with left helicity and negative lepton number to neutrinos with right helicity 27].

As has already been mentioned, in the vicinity of $T_{C}$ only the Standard Model states are sufficiently light to be in thermal equilibrium. Consequently, only coupling of the neutrino to the gauge and Higgs bosons are relevant for the present discussion.

$$
\begin{aligned}
\mathcal{L} & =\frac{g}{2} \frac{m_{\nu}}{M_{W}} \bar{\nu} \nu^{c} H_{0} \\
& -\frac{g}{2 \sqrt{2}} \bar{\nu} \gamma^{\mu}\left(1-\gamma^{5}\right) e W_{\mu}-\frac{\bar{g}}{4} \bar{\nu} \gamma^{\mu}\left(1-\gamma^{5}\right) \nu Z_{\mu}
\end{aligned}
$$

The first term in (39) is responsible for helicity flipping processes, while the second and the third terms contribute to two-body scattering mediated by the light neutrino.

In accordance with the adopted definition of the neutrino lepton number helicity flipping processes 'directly' violate lepton number by two units and washout the neutrino chemical potential. Diagrams with the neutral Higgs exchange are the same (except that now the neutrino is a Majorana fermion) as the ones in Fig. 2 Expressions for the corresponding reduced cross sections and reaction densities are similar to (16) and (17) and to (20) respectively. Analogously, expressions for reduced cross sections and reaction densities of the diagrams with the gauge boson exchange are similar to (22) and (23) and to (24) respectively.
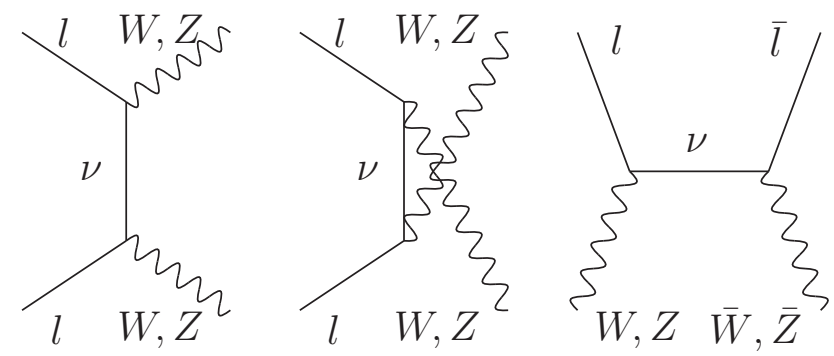

FIG. 4: Lepton number violation in two-body processes.

There are also several diagrams with the light neutrino exchange which violate lepton number by two units. The neutrino chemical potential is washed out 'indirectly' by such processes. Namely, change of the lepton number induced by these processes is later transferred to the neutrino sector by the usual SM processes. Reduced cross sections of the processes (and their charge conjugate) depicted in Fig. 4 are given by

$$
\begin{aligned}
\hat{\sigma}_{s}^{W} & =\frac{g^{4}}{512 \pi}\left(\frac{m_{\nu}}{M_{W}}\right)^{2} \frac{(z-1)^{4}}{3 z^{7}} \\
& \times\left[3 z^{4}+4 z^{3}+58 z^{2}+4 z+3\right] \\
\hat{\sigma}_{t+u}^{W} & =\frac{g^{4}}{16 \pi}\left(\frac{m_{\nu}}{M_{W}}\right)^{2}\left(3-z+z^{2} / 4\right) \\
& \times\left[\sqrt{z(z-4)}+\frac{2}{z-2} \ln \left(\frac{z-2+\sqrt{z(z-4)}}{z-2-\sqrt{z(z-4)}}\right)\right]
\end{aligned}
$$

where $z \equiv s / M_{W}^{2}$. For $T \geqslant m_{W, Z, \ell}$ one can obtain approximate analytical expressions for the corresponding 
reaction densities. To leading order

$$
\begin{aligned}
\gamma_{s}^{W} & =\frac{g^{4} T^{4}}{(4 \pi)^{5}}\left(\frac{m_{\nu}}{M_{W}}\right)^{2}\left(\frac{T}{M_{W}}\right)^{2} \\
\gamma_{t+u}^{W} & =\frac{9 g^{4} T^{4}}{\pi^{5}}\left(\frac{m_{\nu}}{M_{W}}\right)^{2}\left(\frac{T}{M_{W}}\right)^{6}
\end{aligned}
$$

For the neutral current processes $M_{W}$ is to be replaced by $M_{Z}$ and $g$ by $\bar{g}$. In addition, overall coefficients of expressions for the $t+u$ channel reduced cross section $\hat{\sigma}_{t+u}^{Z}$ and reaction density $\gamma_{t+u}^{Z}$ are factor of two smaller than those in (41) and (43).

For ratios of reaction densities to expansion rate of the universe we find

$$
\frac{x \gamma_{s}^{W}}{s H} \sim 10^{-13}\left(\frac{T}{M_{W}}\right)^{2}, \frac{x \gamma_{t+u}^{W}}{s H} \sim 10^{-10}\left(\frac{T}{M_{W}}\right)^{6}
$$

and similar results for the neutral current.

Below the phase transition lepton number violating interactions are clearly too slow to affect the $B-L$ asymmetry generated at the GUT scale, or, equivalently, to set the neutrino chemical potential to zero. Therefore the light Majorana neutrino can be assigned an effective chemical potential, and the rest of the analysis is the same as in the case of leptogenesis with Dirac neutrino.

Baryon and lepton numbers are given by equations (29), while electric charges of different species are given by equations (30). As has been shown in [16, 17] in the leptogenesis with Majorana neutrino scenario the possibility that individual family lepton numbers are much larger than the total lepton number is naturaly realized for certain values of the $\mathrm{CP}$ violating phases. Therefore, terms proportional to $\Delta$ are likely to be important for calculation of the lepton and baryon asymmetries.

\section{SUMMARY.}

In this paper lepton and baryon number asymmetries in two scenarios of leptogenesis have been considered.

It has been pointed out that if the sphalerons are in thermal equilibrium, then the $3 B+L$ sum is zero for left-handed fermions.
Despite the fact that a Majorana fermion does not carry conserved quantum numbers and does not have a chemical potential, in the early universe the rate of the processes which bring number of neutrinos with different chirality to equilibrium is much smaller than expansion rate of the Universe, and therefore the neutrino can be assigned an effective chemical potential.

In the scenario of leptogenesis with Dirac neutrinos the smallness of the neutrino mass assures that lepton number asymmetries stored in the left- and right-handed neutrinos do not equilibrate. The right-handed neutrinos carry only a small fraction of the Universe energy density and have very little impact on the effective number of relativistic degrees of freedom, constrained by the BBN.

Thus the two scenarios are similar from the cosmological point of view and yield the same relation among the initial lepton and the final baryon asymmetries.

Above the phase transition baryon and lepton numbers are proportional to the total initial $B-L$ asymmetry. Number of the down quarks exceeds number of the up quarks and total electric charge of the leptons is positive.

Below the phase transition due to the mass effects the final baryon number is nonzero even if the initial $B-L$ asymmetry is zero, provided that the individual lepton numbers $L_{i}$ are sufficiently large. As the oscillations equalize chemical potentials of the neutrinos long before the BBN epoch, this possibility is consistent with the BBN constraints on the effective number of relativistic degrees of freedom.

If $B-L$ is large in comparison with $\Delta$ then there is an excess of down quarks and total electric charge of the leptons is positive immediately after the phase transition. On the contrary, if total $B-L$ is zero, there is an excess of up quarks and total charge of the leptons is negative.

\section{Acknowledgments}

I would like to thank Professor E A Paschos for inspiration and useful discussions. Financial support from the Graduiertenkolleg 841 "Physik der Elementarteilchen an Beschleunigern und im Universum" at University of Dortmund is gratefully acknowledged.
[1] A. D. Sakharov, JETP Letters 5, 24 (1967).

[2] G. 't Hooft, Phys. Rev. Lett. 37, 8 (1976).

[3] M. Fukugita and T. Yanagida, Phys. Lett. B174, 45 (1986).

[4] K. Dick, M. Lindner, M. Ratz and D. Wright, Phys. Rev. Lett. 84, 4039 (2000), hep-ph/9907562.

[5] J. A. Harvey and M. S. Turner, Phys. Rev. D42, 3344 (1990).

[6] H. K. Dreiner and G. G. Ross, Nucl. Phys. B410, 188 (1993), hep-ph/9207221.

[7] V. A. Rubakov and M. E. Shaposhnikov, Usp. Fiz. Nauk
166, 493 (1996), hep-ph/9603208.

[8] E. W. Kolb and M. S. Turner, Redwood City, USA: Addison-Wesley (1990) 547 p. (Frontiers in physics, 69).

[9] M. J. Savage, R. A. Malaney and G. M. Fuller, Astrophys. J. 368, 1 (1991).

[10] A. D. Dolgov et al., Nucl. Phys. B632, 363 (2002), hep-ph/0201287.

[11] S. H. Hansen, G. Mangano, A. Melchiorri, G. Miele and O. Pisanti, Phys. Rev. D65, 023511 (2002), astro-ph/0105385.

[12] S. Burles, K. M. Nollett, J. N. Truran and M. S. Turner, 
Phys. Rev. Lett. 82, 4176 (1999), astro-ph/9901157.

[13] S. Pakvasa and J. W. F. Valle, Proc. Indian Natl. Sci. Acad. 70A, 189 (2004), hep-ph/0301061.

[14] M. Maltoni, T. Schwetz, M. A. Tortola and J. W. F. Valle, New J. Phys. 6, 122 (2004), hep-ph/0405172.

[15] L. Bento and F. C. Santos, Phys. Rev. D71, 096001 (2005), hep-ph/0411023.

[16] T. Endoh, T. Morozumi and Z.-h. Xiong, Prog. Theor. Phys. 111, 123 (2004), hep-ph/0308276.

[17] T. Fujihara et al., Phys. Rev. D72, 016006 (2005), hep-ph/0505076.

[18] M. Laine and M. E. Shaposhnikov, Phys. Rev. D61, 117302 (2000), hep-ph/9911473.

[19] An analysis applicable also in the $T \gg \phi$ limit, where $\phi$ is the temperature dependent Higgs expectation value, has been performed in [18]

[20] For instance, in extensions of the standard model based on the $E_{6}$ gauge group each generation contains two Higgs doublets. Supersymmetric extension of the SM necessarily contain at least two Higgs doublets responsible for masses of the up and the down quarks. However, if quarks of given charge are coupled to more than one light Higgs, strong flavor-changing neutral currents arise. Numerical values are given here only for $m=1$.

[21] In the case of the standard model charged components of the Higgs doublet are eaten up by the $W$ and $c_{-}$is identically zero below the phase transition.

[22] The created object is a $S U_{L}(2)$ singlet because both upand down components of weak doublets are created out of the vacuum and a color-singlet because quarks of all the three colors are created simultaneously.

[23] Note that although the individual lepton numbers $L_{i}$ are not conserved, the difference $L_{i}-L / N$ is conserved by the sphalerons.

[24] If the phase transition is strongly first order, then the anomalous electroweak transitions switch off immediately after the phase transition. In this case, obviously, the baryon number is given by its value above the phase transition.

[25] It is easy to see, that if $\mu_{i}=0$ below the electroweak phase transition, then from the electric charge conservation and relation (12), enforced by the sphaleron processes, it follows that $\mu_{u L}=\mu_{W}=0$ and therefore baryon and lepton numbers are zero.

[26] Note, that above the EW phase transition lepton number asymmetry is partially washed out in the scattering processes mediated by the heavy Majorana neutrino which violate lepton number by two units. However, these processes are effectively frozen out already at temperatures $\sim 0.1 M_{N}$. Given the current estimates of the righthanded neutrino mass $M_{N} \sim 10^{10}-10^{12} \mathrm{GeV}$ we conclude that the washout processes are irrelevant in the vicinity of $T_{C}$.

[27] For a massive particle helicity is not invariant under Lorentz transformations. However, in an isotropic Universe the comoving thermal bath frame is a priveleged frame where isotropy enforces the spin density matrix to be diagonal in the helicity basis. 